\section{Promoting the Safety of Healthcare Workers in Africa: From HIV Pandemic to Ebola Epidemic}

To the Editor-Healthcare workers (HCWs) are at risk of blood contact and thus of contamination by bloodborne pathogens. The HIV pandemic has brought about an awareness of this risk. Most epidemiological data on occupational exposure to blood and other body fluids are from studies conducted in North America and in Europe. Studies in Africa indicate that exposure is frequent, rarely reported, and at high risk of conveying occupational infection in the working environment (eg, due to insufficient risk awareness and lack of adequate equipment) and high population prevalence of bloodborne infections (ie, HIV and hepatitis viruses). ${ }^{1}$ Moreover, venous blood sampling seems to be the most frequent invasive procedure related to needle stick injuries (NSI) and the one that involves the highest risk of occupational infection. ${ }^{2}$ While effective measures of prevention do exist, ${ }^{3,4}$ the protection of HCWs rarely seems to be a priority, and the problem of infectious risks linked to venous sampling remains a worry for those working in medical facilities in West Africa. Furthermore, an increase in invasive procedures following extension of disease management and access to care makes this situation all the more acute in programs focusing on specific diseases like HIV/AIDS. ${ }^{5}$

Although risks of contamination with the Ebola virus are far more intense than those with HIV, the current Ebola outbreak in West Africa, like several of the previous outbreaks, illustrates this situation by the deadly toll that has resulted from infected caregivers, accounting for $5 \%$ to $10 \%$ of victims. In-hospital transmission to other patients and their relatives, who often participate in care in hospital wards, further accelerates Ebola outbreaks. ${ }^{6}$ It has been noted repeatedly throughout the history of Ebola outbreaks that substandard hospital infection control practices contribute to making HCWs both time vectors and victims of the epidemic. ${ }^{7}$

In the framework of a partnership between Niamey's National Hospital (NNH) and 3 Paris Public Hospitals initiated in 2006 and coordinated by the Ensemble pour une Solidarité Thérapeutique en Réseau (ESTHER), the Group for the Prevention of Occupational Infections in Healthcare Workers (GERES) has developed a program specifically devoted to the protection of NNH HCWs. Since 2010, this action has been part of the African Partnership for Patient Safety (APPS), a program launched in 2008 by the World Health Organization (WHO) Regional Office for Africa to improve the quality of care and patient safety.

The program's first steps included setting up a local committee for the coordination of prevention efforts, the implementation of a survey to document occupational exposures, and the development of procedures allowing quick access to treatment in case of exposure.

Procedures at high risk of NSI were identified, and a seroprevalence survey of HBV and HCV infection was conducted in 2009 among NNH personnel. ${ }^{8}$ Following these studies, nonimmune HCWs were offered HBV vaccination and currently infected HCWs were offered treatment; safety devices for venous sampling were introduced in July 2013 under funding from APPS in 7 targeted hospital departments. Ongoing surveillance of blood-borne exposures showed that no more NSIs had been reported in relation to venous sampling in these departments since the safety devices were introduced. In late November 2013, 102 of the 450 HCWs at the NNH were randomly selected and questioned, on a given day, using a selfreporting questionnaire. Approximately $50 \%$ of the sample ( 55 of 102) used the safety device introduced in July 2013; $97 \%$ of HCWs feared the risk of HIV infection; and $81 \%$ feared the risk of tuberculosis. Among the responding HCWs at the hospital, $66 \%$ were aware of the risk prevention measures taken there; $50 \%$ believed that these measures needed to be improved; and 93\% thought that the health authorities should do more to protect HCWs. All users of safety devices (100\%) believed that these devices reduced the risk of occupational exposure; $87 \%$ reported that they were easier to use than a syringe for blood drawing; $76 \%$ reported that they were faster; $76 \%$ reported that they were easy to learn; $89 \%$ reported that they were reassuring when caring for an HIV-infected patient; and 98\% wanted to continue to use them. In parallel with actions taken at the $\mathrm{NNH}$, a guide to implementing an effective program for the prevention of accidental blood exposure in healthcare settings in French-speaking African countries was co-authored by French and African experts with support from ESTHER. ${ }^{9}$ Our partnership with the NNH is continuing with a program to prevent the risk of transmission of tuberculosis at the NNH. The years of partnership have shown that it is possible for a hospital in Sub-Saharan Africa to engage in a risk-reduction program that seems to motivate HCWs and to improve their well-being.

The development of the management of PLHIV in SubSaharan Africa has made it essential to support healthcare professionals caring for HIV-infected patients, enabling them to provide care while minimizing risk of HIV transmission. ${ }^{10}$ Today, some 30 years after the appearance of the first cases of HIV/AIDS and 10 years after cheaper generic antiretroviral drugs became available in Africa, venous sampling is still not safe in most healthcare settings and also lacks basic personal protective equipment and sharp disposal containers. The protection of medical personnel against infectious risks is, however, the responsibility of the authorities, of national policy makers, of directors of medical healthcare facilities. And as was the case for antiretroviral drugs, there is an urgent need to reach agreements enabling provision of inexpensive, 
safe equipment for HCWs in Africa and in resource-limited settings. The current Ebola outbreak in Africa emphasizes the urgent need for such developments.

\section{ACKNOWLEDGMENTS}

Financial support: Financial support for the program conducted at NNH was provided by ESTHER, a French Public Agency that promote access to care for people living with HIV in developing countries. Additional support for this action was provided by the African Partnership for Patient Safety.

Potential conflicts of interest: All authors report no conflict of interest relevant to this letter.

Author contributions: All authors made substantial contributions to the implementation of the program of action, to the collection of data, or to the writing of the letter. All authors approved the submitted version of the letter.

\author{
Elisabeth Rouveix, $\mathrm{MD} ;{ }^{1,4,5}$ \\ Boubakar Madougou, MD; ${ }^{7,8}$ \\ Gérard Pellissier, $\mathrm{PhD}{ }^{1}$ \\ Hama Diaougah, $\mathrm{MD}^{7}$ \\ Sahada Moussa Saley, $\mathrm{MD}^{7}$ \\ Pierre de Truchis, MD; ${ }^{4,6}$ \\ Dionké Fofana, PJM; ${ }^{3}$ \\ Isabelle Lolom, MSc; ${ }^{1,2}$ \\ Jean-Baptiste Brunet, $\mathrm{MD}^{1}$ \\ Elisabeth Bouvet, $\mathrm{MD}^{1,2}$
}

Affiliations: 1. Group for the Prevention of Occupational Infections in Healthcare Workers (GERES), Bichat Faculty of Medicine, Paris VII University, Paris, France; 2. Infection Control Unit and Department of Infectious Diseases, Bichat Hospital, APHP, Paris, France; 3. Ensemble pour une Solidarité Thérapeutique en Réseau, Paris, France; 4. Entraide Santé 92, Vaucresson, France; 5. Department of Medicine, Ambroise Paré Hospital, APHP, Boulogne-Billancourt, France; 6. Department of Infectious Diseases, Raymond Poincaré Hospital, APHP, Garches, France; 7. Department of Medicine, Niamey's National Hospital, Niamey, Niger; 8. Ensemble pour une Solidarité Thérapeutique en Réseau, Niamey, Niger.

Address correspondence to G. Pellissier, GERES, UFR de Médecine Bichat, 16 rue Henri Huchard, F-75018 Paris, France (gerard.pellissier@geres.org). Infect Control Hosp Epidemiol 2015;36(3):361-362

(c) 2015 by The Society for Healthcare Epidemiology of America. All rights reserved. 0899-823X/2015/3603-0020. DOI: 10.1017/ice.2014.68

\section{REFERENCES}

1. Lee R. Occupational transmission of bloodborne diseases to healthcare workers in developing countries: meeting the challenges. J Hosp Infect 2009;72:285-291.

2. Tarantola A, Koumaré A, Rachline A, et al. A descriptive, retrospective study of 567 accidental blood exposures in healthcare workers in three West African countries. J Hosp Infect 2005;60: 276-282.

3. Lamontagne F, Abiteboul D, Lolom I, et al. Role of safetyengineered devices in preventing needlestick injuries in 32 French hospitals. Infect Control Hosp Epidemiol 2007;28:18-23.

4. Alvarado-Ramy F, Beltrami EM, Short LJ, et al. Comprehensive approach to percutaneous injury prevention during phlebotomy: results of a multicenter study, 1993-1995. Infect Control Hosp Epidemiol 2003;24:97-104.
5. Harries AD, Zachariah R, Tayler-Smith K, et al. Keeping health facilities safe: one way of stengthening the interaction between disease-specific programs and health systems. Trop Med Int Health 2010;15:1407-1412.

6. Baron RC, McCormick JB, Zubeir OA. Ebola virus disease in southern Sudan: hospital dissemination and intrafamilial spread. Bull World Health Org 1983;61:997-1003.

7. Muyembe-Tamfum JJ, Kpasa M, Kiyungu C, Colebunders R. Ebola outbreak in Kikwit, Democratic Republic of the Congo: discovery and control measures. J Inf Dis 1999;179:S259-S262.

8. Pellissier G, Yazdanpanah Y, Adehossi E, et al. Is universal $\mathrm{HBV}$ vaccination of healthcare workers a relevant strategy in developing endemic countries? The case of a university hospital in Niger. PLOS ONE 2012;7:e44442. doi:10.1371/Journal.pone. 0044442.

9. GERES (Groupe d'Etude sur le Risque d'Exposition des Soignants aux agents infectieux), ESTHER (Ensemble pour une Solidarité Thérapeutique en Réseau). Prévention et prise en charge des AES. Manuel pratique. Document à l'attention des établissements de soins d'Afrique francophone, February 2008, 115 pp. GERES website. http://www.geres.org/docpdf/manuelaesgeres08.pdf. Published 2008. Accessed October 22, 2014.

10. Reis C, Heisler M, Amowitz LL, et al. Discriminatory attitudes and practices by health workers toward patients with HIV/AIDS in Nigeria. PLoS Med 2005;2:0743-0752.e246.

\section{Veterans Affairs Medical Center Employee Comments Suggest Additional Educational Targets to Improve Influenza Vaccination Campaigns}

To the Editor-The Veterans Health Administration strongly encourages all employees to receive an annual influenza vaccine, with the goal of achieving a $90 \%$ vaccination rate among healthcare personnel (HCP) by $2020 .^{1}$ A nationwide survey conducted by Schult et al. queried reasons that Veterans Affairs employees did not get the 2009-2010 influenza vaccine, offering 12 specific reasons for declining the vaccine. ${ }^{2}$ Interactions with employees at two Veterans Affairs Medical Centers (VAMCs) raised the possibility of a wider array of reasons for vaccine refusal. We surveyed employees at both VAMCs regarding their reasons for accepting or declining the influenza vaccine in the 2013-2014 season, including the option to explain their views using comments entered as free text.

The institutional review boards at both participating facilities reviewed and approved the survey and study design. Employees at two VAMCs were invited via e-mail to participate in an anonymous, voluntary survey, accessed through an Internet link, that took $<5$ minutes to complete (Qualtrics, Provo, UT). The survey included questions similar to those 